María Ángeles Alcaraz Ariza* \&

Françoise Salager-Meyer*

\title{
Género y crítica en la prosa médica escrita en español: Función comunicativa y relación de poder ${ }^{1}$
}

\begin{abstract}
This study addresses the issue of academic conflict (AC) in four different genres of medical discourse, viz., editorials, review and research papers and case reports. The rhetorical strategies used to convey $\mathrm{AC}$ were recorded and their qualitative features (their level of commitment or detachment, their overall tone, the presence of irony, sarcasm, etc.) were analyzed in 40 medical articles written in Spanish in the last decade of the 20th century. Our results show that the communicative function of each genre and the rank/status power relations that exist between authors and their audience have a direct bearing on the way medical researchers express their dissension.
\end{abstract}

Keywords: academic conflict, Spanish medical discourse, genre studies

\section{Introducción}

En las dos últimas décadas varios son los estudios que han versado sobre «la retórica de la confrontación académica». Si algunos autores han abordado este tema desde una perspectiva general (Myers 1989; Swales 1990; North 1992; Belcher 1995; Schramm 1996; Vihla 1999; Hemais 2001),

1 La presente investigación ha sido posible gracias a las siguientes ayudas: Beca $\mathrm{n}^{\mathbf{0}}$ POSTOO-09-80 de la Dirección General de Enseñanzas Universitarias e Investigación de la Generalitat Valencia (Valencia, España), Subvención M-657-99 del Consejo de Desarrollo Científico, Humanístico y Tecnológico de la Universidad de Los Andes (Mérida, Venezuela) y Subvención S1-98003578 del Consejo Nacional de Investigaciones Científicas (CONICIT) de Venezuela.

* María Ángeles Alcaraz Ariza

Facultad de Filosofía y Letras

Departamento de Filología Inglesa

Universidad de Alicante

E-Alicante
* Françoise Salager-Meyer

Escuela de Posgrado. Facultad de Medicina y Escuela de Idiomas Modernos Facultad de Humanidades y Educación Universidad de Los Andes V-Mérida 
otros lo han hecho desde un enfoque cuantitativo (Chubin y Moitra 1975; Moravcsik y Murugesan 1975). Tampoco han faltado estudios interdisciplinares (Hunston 1993; Swales y Feak 1995; Kourilova 1996; Motta-Roth 1998), interlingüísticos e interculturales (Nguyen 1988; Do 1989; Taylor y Chen 1991; Bloch y Li 1995; Ahmad 1997; Duszak 1997; Farrell 1997) o diacrónicos e interculturales (Salager-Meyer 1999, 2000; Salager-Meyer y Zambrano 2001). Todos estos estudios han ayudado a aclarar nuestra comprensión del conflicto académico en cierta medida. Sin embargo, como bien señala Hyland (2000: 45):
Most of the research on compliments and criticisms has occurred within a framework based on politeness, has examined speech and focused on complimenting behavior ... but there is little work on how this may vary in particular genres and contexts. ${ }^{2}$

Por otra parte, todas las investigaciones mencionadas se han centrado en el discurso científico escrito en alemán, checo, chino, inglés, francés, malayo, polaco y vietnamita, y se echan en falta estudios de una lengua como el español que, a pesar de contar con 400 millones de hablantes nativos y ser la tercera lengua más hablada del mundo, ha merecido escasa atención por parte de los lingüistas. Por ello es nuestro propósito intentar remediar esta situación, y en este sentido debemos mencionar que el presente trabajo se inscribe en una investigación ya iniciada (Salager-Meyer y Alcaraz Ariza 2001, 2002), que aspira a complementar los resultados de los estudios referidos.

\section{Objetivos}

Antes de especificar el objetivo del presente estudio, es preciso definir de forma clara y precisa el concepto de «confrontación académica»o «controversia académica» (Zambrano 1999: 13) — también referida como professional disagrement — (Hunston 1993: 116) o «academic conflict» (Kourilova 1996: 20). Por «confrontación académica» se entiende toda expresión, estrategia o formulación lingüístico-retórica utilizada por los científicos para manifestar su desacuerdo con opiniones/juicios previa-

2 De hecho, la noción de «género» ha sido primordial en los análisis del lenguaje profesional llevados a cabo por Swales (1990), Bhatia (1993), Paltridge (1997) y Bazerman (1998), además del propio Hyland (2000). 
mente emitidos o con los resultados/conclusiones presentados por otro(s) miembro(s) de la comunidad científica a la cual pertenecen. Este concepto está íntimamente relacionado con el de «reivindicación académica»o proposición que los científicos hacen a los conocimientos adquiridos por la comunidad de la cual forman parte. Una confrontación académica (CA) debe, pues, entenderse en el sentido de conflicting knowledge claim (Hunston 1993: 109), ya que generalmente incluye comentarios negativos acerca de una investigación publicada anteriormente (la crítica apunta hacia el producto) o sobre el(los) autor(es) de dicha investigación (el blanco de la crítica es entonces el destinatario).

Ahora bien, nuestro objetivo principal en este estudio consiste en analizar, desde un punto de vista cualitativo, la controversia académica en el contexto particular de la función comunicativa de cuatro diferentes géneros ${ }^{3}$ del discurso biomédico escrito en español (Editoriales, Artículos de Revisión, Artículos Originales y Notas Clínicas) en el periodo que abarca la última década del siglo XX (1990-1999) ${ }^{4}$.

Además, y en consonancia con los últimos estudios sobre el concepto de «género» según los cuales se trata de constructos sociales (Freedman y Midway 1994), otro de nuestros objetivos consiste en relacionar las variaciones observadas (si las hay) con los siguientes rasgos sociopragmáticos y/o socio-discursivos: 1) la función comunicativa de cada

3 «Tipo de texto», «género» o «subgénero»? Al parecer todavía no se ha alcanzado un consenso en cuanto a la diferencia existente entre «tipo de texto» y «género». Bailar y otros (1984), al igual que Álvarez Dardet y otros (1987), hablan de «tipos de texto». Biber (1988) opina que «género» y «subgénero» pueden aplicarse a clases basadas en criterios externos, mientras que «tipo de texto» puede usarse para referirse a agrupamientos basados en la forma linguística. Swales (1990), por su parte, define el género como un tipo de texto determinado por el cometido, el entorno y el propósito comunicativo. Para algunos escritores, como Devitt (1991) y Dudley-Evans y St John (1998), ambos términos son sinónimos, mientras que Connor (1996: 11) considera que el género refers to texts formed according to cultural and traditional expectations as required by specific purposes and tasks such as a research report in biology. Grabe y Kaplan (1997) se refieren a «tipos de texto» y Bhatia (1998: 21) opina que a survey article, a review article, a state-of-the art article can be distinguished as sub-genres of what is popularly known as the research article, al igual que Posteguillo (2000) que alude al artículo original como el género estudiado con más frecuencia. Otros autores que también utilizan el término «género» son Salager-Meyer (2000) y Ferguson (2001), y en este artículo seguiremos su propuesta.

4 En estos momentos estamos realizando un análisis cuantitativo de las CCAA registradas en nuestra muestra. 
género; 2) el nivel de la reivindicación de conocimiento propio de cada género ya que, según Kress (1986: 112), each specific genre encodes different power relations between writer/speaker; 3 ) el rango/estatus de las relaciones de poder existentes entre los autores y su público así como las expectativas de este último.

En resumen, en este estudio intentaremos contestar las preguntas siguientes:

1) ¿En qué medida las estrategias retóricas utilizadas por los médicos de habla hispana para expresar su desacuerdo reflejan su compromiso u objetividad en los cuatro géneros investigados?

2) ¿Cómo se puede explicar dicho compromiso en términos de la función comunicativa propia de cada género, es decir, el papel que cada género desempeña en el marco de la comunidad médica investigadora?

\section{Muestra lingüística}

Basándonos en el hecho de que los avances científicos y educativos en el campo de la medicina se canalizan principalmente a través de publicaciones periódicas - según señalan diversos investigadores que se han ocupado del tema, entre otros Fye (1987), López Piñero y otros (1989) y López López (1996) -, hemos optado por recurrir a artículos de revista (y no secciones de libros) como fuente primaria de estudio. Hemos entonces utilizado para la confección de nuestro corpus diversas revistas médicas españolas (Anales de Medicina Interna, Anales Españoles de Pediatría, Atención Primaria, Medicina Clínica, Medicina Intensiva, Revista Clínica Española) publicadas durante la década 1990-2000 y de las cuales hemos seleccionado al azar un total de 40 artículos. De acuerdo con los cuatro géneros más característicos de los textos médicos profesionales, estos 40 artículos se componen de 10 Editoriales, 10 Artículos de Revisión (incluidos metanálisis 5 ), 10 Artículos Originales y 10 Notas Clínicas.

La asignación de categoría de género no ha revestido ninguna dificultad puesto que el género de los artículos seleccionados se especifica clara-

5 En el metanálisis se analizan los datos procedentes de diferentes estudios desde una perspectiva cuantitativa con el fin de incrementar el tamaño de la muestra y así reducir la incertidumbre de los resultados. 
mente, tanto en la portada de cada revista como en el encabezamiento de los artículos. Podemos, por tanto, considerar que son todos ellos prototypical exemplars (Swales 1990: 52) de cada género6 ${ }^{6}$ Los artículos revisados han sido extraídos de revistas disponibles en la hemeroteca del Hospital General Universitario de Alicante (Alicante, España). La selección de estas revistas se ha realizado mediante la consulta del registro que la hemeroteca de dicho hospital lleva sobre las revistas españolas más consultadas en la actualidad por los investigadores médicos que acuden a dicho Centro. Los destinatarios de estos artículos son miembros de la comunidad médica con diferentes niveles de competencia profesional (facultativos, investigadores y estudiantes de medicina).

Con el fin de que el código lingüístico utilizado en nuestro corpus fuera representativo del español escrito normativo o estándar, sólo hemos seleccionado artículos cuyos autores tuvieran apellidos españoles y desempeñaran su labor en hospitales, universidades o instituciones españolas. Por último, quisiéramos señalar que para poder obtener una visión más precisa de la CA en nuestra muestra, hemos analizado cada artículo en su totalidad en lugar de ceñirnos a una longitud predeterminada o a una sección específica del mismo.

\section{Método}

La presente investigación se ha desarrollado en dos fases:

1) Puesto que estamos interesados en obtener un repertorio de las estrategias retóricas (formales) utilizadas por los científicos españoles para formular sus críticas, en cada uno de los 40 artículos que conforman la muestra estudiada hemos buscado y registrado manualmente todas las realizaciones lingüísticas de las proposiciones que reflejan una discrepancia con las reivindicaciones de conocimiento presentadas anteriormente por científicos individuales o por la comunidad científica como ente colectivo.

2) Hemos clasificado estas CCAA en dos grandes categorías de acuerdo con la fuerza ilocucionaria del acto de habla de la CA, es decir,

6 La noción de «prototipo» es primordial en los estudios sobre géneros puesto que se refiere al modo en cómo determinadas propiedades (función comunicativa, forma, estructura y expectativas de la audiencia) operate to identify the extent to which an exemplar is prototypical of a particular genre (Swales, 1990: 52). 
según la presencia o ausencia de elementos matizadores en su formulación lingüística. Esta distinción corresponde a la diferencia que Chafe (1982: 48) establece entre involvement y detachment, y Vassileva (2001: 83) entre committment y detachment:

a) CA directa (crítica formulada sin desvío, abiertamente), en la cual el autor se responsabiliza enteramente de su acto crítico (ejemplo 1):

1. «Al analizar estas técnicas de localización, algunos autores observan que unas pruebas complementan a otras en los hallazgos, obteniendo una excelente rentabilidad conjunta (*). Sin embargo, esta conclusión es falaz pues, a la hora de tomar una decisión quirúrgica con datos discordantes, ¿qué exploración está ofreciendo el resultado correcto?» 7

b) CA indirecta (crítica formulada de manera encubierta, velada o disfrazada), en la cual el autor utiliza elementos modalizadores para suavizar o mitigar la intensidad de su desacuerdo. Esta CA se suele manifestar de dos modos distintos:

- mediante el uso de matizadores tradicionales (ejemplo 2);

- mediante la ocultación del agente de la crítica, cuyo peso recae sobre hechos inanimados que se convierten en los autores de la CA (ejemplo $3)$.

2. «Sin embargo, el no expresar los datos corregidos en tasa por 100.000 habitantes puede conllevar a la falsa creencia de que los ancianos en los países en vías de desarrollo están relativamente libres de padecer la enfermedad.»

3. «La elevada prevalencia de deficiencia de Proteína Co de Proteína $S$ halladas por D’Angelo y Cols. (*) o Sacco y cols. (*) respectivamente no se confirma en nuestro estudio.»

El enfoque adoptado en este estudio se basa en un análisis textual. Como este tipo de enfoque implica que un solo lector lee e interpreta los textos,

7 En todos los ejemplos las palabras/expresiones que reflejan la CA directa aparecen en cursiva y la CA indirecta se ha subrayado. El símbolo * indica que el ejemplo seleccionado contiene un número entre paréntesis o en superíndice, indicador de una referencia bibliográfica. En nuestra transcripción de los ejemplos hemos respetado fielmente la ortografía y puntuación originales. A pesar de que los ejemplos presentados en ese artículo han sido sacados de su contexto original, confiamos en que sean lo bastante explícitos como para ilustrar nuestros argumentos. 
a menudo se objeta que otros analistas podrían sacar conclusiones diferentes, y que se trata, por tanto, de un análisis subjetivo. Con el fin de contrarrestar esta objeción y dar mayor validez a la interpretación de nuestros descubrimientos, hemos hecho nuestra la observación de Valle (1999: IX), según la cual a project in which the study is outside the writer's own discipline necessarily requires help from members of the scientific community under study. En consecuencia, hemos solicitado el asesoramiento de un «informador especializado» (specialist informant), práctica por otra parte muy recomendada en todas las variedades de análisis de la prosa académica (Widdowson 1983; Huckin y Olsen 1984; Trimble 1985; Hutchinson y Waters 1987).

\section{Resultados y discusión \\ 5.1. Editoriales (ED)}

El ED es un artículo breve en el que se expresa una opinión o se interpretan los hechos u opiniones de otros (Mulrow 1987; Pulido 1987). Los 10 Editoriales de nuestro corpus tienen una longitud que oscila entre 1000 y 3000 palabras y cinco de ellos vienen firmados por dos autores, mientras que los cinco restantes los firma un solo autor. En lo que se refiere a su estructura, los Editoriales se organizan libremente, según el criterio de cada autor. No suelen ir acompañados de tablas o figuras y las referencias bibliográficas no son por lo general muy numerosas.

A veces los Editoriales exponen el comentario sobre un Artículo Original publicado en el mismo número de la revista. En nuestra revisión sólo tres de ellos cumplen esta característica, pero no para censurarlos sino para formular algunas recomendaciones, lo que, en cierto modo, es comprensible porque ello equivaldría a poner en entredicho el comité de árbitros que ha aceptado el Artículo Original para su publicación. En los siete Editoriales restantes, las CCAA son muy frecuentes y en en seis de ellos se caracterizan por su tono directo, agresivo, mordaz irónico y/o sarcástico. Como botón de muestra, mencionemos el ED titulado «Guías de práctica clínica basadas en la evidencia». Las CCAA vertidas en él destacan especialmente por su tono ácido, imperativo y provocador, $\mathrm{y}$ sin duda habrán estimulado a los lectores emotiva e intelectualmente y tal vez hayan sido origen de sustanciosas reacciones de controversia y debate en las páginas de «Correspondencia» $\mathrm{o}$ «Cartas al Director» de la 
misma revista o de otras de la competencia. Basta indicar que este ED se inicia con una cita extraída de la obra titulada «Sofismas y desatinos en medicina» (Skrabanek y McCormick 1992), que reza como sigue:

«Escepticemia: trastorno raro y generalizado de baja infectividad. La educación recibida en las facultades de medicina puede llegar a conferir inmunidad de por vida frente a ella». ${ }^{8}$

Esta cita, en la cual la ironía y el sarcasmo afloran por doquier ${ }^{9}$, sirve al autor para lanzar un ataque sin cuartel en el que no deja títere con cabeza. Arremete contra la irrefutabilidad del principio de autoridad, las obsoletas enseñanzas y prácticas médicas, la falta de autocrítica de muchos miembros de la profesión, el proceso sanitario, la inadecuada metodología estadística y la deficiente redacción de protocolos y programas. Todas estas críticas las deja bien a las claras el largo párrafo, en realidad una retahila de reprobaciones, que reproducimos a continuación, a pesar de su longitud, y que bien merecería por sí solo un análisis más detallado:

4. «Históricamente la enseñanza de la medicina, de pre y posgrado se ha impartido y se sigue impartiendo en unos ambientes de majestuosidad y autoridad difíciles de cuestionar. La crítica, la autocrítica, la duda y la refutación nunca fueron parte de nuestra formación. Tampoco se nos enseñó en esos "sacrosantos lugares" metodología científica, lectura crítica de investigaciones, metaanálisis, etc. En cambio, nuestra formación giró en torno a autores, escuelas, textos y documentos claves; y que se esperaba que nunca cuestionásemos. En resumen, era más importante quién decía que lo que decía. Un aspecto, que en los últimos años se incorpora a la práctica clínica, es la epidemiología-estadística. Esta incorporación es positiva siempre y cuando, como cualquier herramienta, se utilice de manera correcta y para fines loables. Pero como todo en la vida depende de las manos en quien cae.

8 "Scepticaemia": a rare and generalized low infectivity disorder. The education received in our medical schools may succeed in bestowing life-long immunity.

9 Testigos de la ironía y del sarcasmo serían, a nuestro parecer, no sólo el uso del vocablo «escepticemia», término inexistente que probablemente se ha formado a partir de un cruce ingenioso entre «e(scepticismo)» 'incredulidad o duda acerca de la verdad o eficacia de alguna cosa' y «septicemia» 'grupo de infecciones generales graves debidas a la penetración, de forma continua o periódica, de gérmenes patógenos en la sangre circulante, originando focos a distancia', sino también con la unión de los adjetivos antagónicos «raro» y «generalizado». ; Y esta enfermedad imaginaria la vence la enseñanza impartida en las facultades de medicina! 
Hay quien usa estos útiles como los borrachos las farolas para apoyarse y no para que les alumbren el camino. Cuanto más aparato estadístico-epidemiológico y más complejo es éste, más se sustentan las afirmaciones que se hacen. Además por la "formación-deformación" que tenemos los profesionales sanitarios, las nuevas aportaciones o correcciones a los conocimientos previos se incorporan apresuradamente a la práctica clínica cotidiana, con lo que ello implica.» ${ }^{10}$

En este texto, además del sarcasmo recurrente ${ }^{11}$, se aprecia una escritura comprometida, reflejada en el uso de pronombres y verbos flexionados en primera persona («nuestra», «nos», «cuestionásemos», «tenemos») utilizados con intención generalizadora, puesto que the first person plural pronoun may be used to indicate that the writer and reader belong to the same 'peer' group (Vihla 1999: 85).

Si en este párrafo se atacan las autoridades académicas, creemos que el ejemplo siguiente tomado del mismo ED deja entrever cierta posición ideológica que trasciende la mera argumentación crítica y que no sólo visa a las referidades autoridades, sino también a estamentos gubernamentales:

5. «El principal escollo con el que se encuentra la medicina basada en la evidencia, y por ende las guías de práctica clínica basadas en la evidencia, no es otro que el inmovilismo más conservador de ciertas personas e instituciones que quieren impedir cualquier intento de avance que se aleja de los cánones clásicos.»

Siguiendo con el mismo ED, en otro ejemplo el autor se atreve incluso a criticar un ED publicado en una prestigiosa revista de fama internacional como The Lancet, aunque sin mencionar el nombre del autor de dicho ED, ni siquiera en la bibliografía:

10 Translation of central parts of citation: Nor in those "sacrosanct" places have we been taught scientific methodology, research critical reading, meta-analysis, etc. On the contrary, our training focused on key authors, schools, texts and documents which we were not expected to question or discuss. In other words, the most important thing was who was saying what rather than what was being said. ... Everything in life depends on whose hands any tool (e.g., statistical epidemiology) falls into. ... Some use such tools like drunkards use streetlamps to lean on rather than to light up their way.

$11 \mathrm{Al}$ sarcasmo contribuyen las comparaciones que se hacen: las facultades de medicina se califican de «sacrosantos lugares» (y son por tanto intocables), la estadística, amén de compleja, no se utiliza para su fin primario, al igual que tampoco las farolas para las personas bebidas, etc. 
6. «Una muestra de lo que acabo de decir es el editorial de la revista The Lancet del 23 de septiembre de 1995, y que lleva por título «Evidence-based medicine, in its place» (La medicina basada en la evidencia en su sitio).... Nunca llegaremos a saber si en dicho editorial hay más "pelusa" que inmovilismo, o viceversa.»12

En otro ED que versa sobre las aplicaciones terapéuticas actuales de la vitamina $\mathrm{C}$, las marcas de contenido negativo presentes en vocablos disfóricos e intensificadores («proliferación», «presuntas», «sonados fracasos», «descrédito») y el tono sarcástico incluido en la cláusula adverbial litótica «no poco» son la antesala de la máxima con la que el autor cierra su crítica:

7. «Esta proliferación de presuntas aplicaciones de la vitamina $C$, unida a los sonados fracasos en la demostración de eficacia clínicamente relevante en el resfriado común* y en el cáncer avanzado*, han contribuido no poco a su descrédito (lo que sirve para casi todo, habitualmente no sirve para casi nada).»13

A este mismo ED pertenece el siguiente ejemplo, en el cual la CA se ha mitigado mediante el uso del semi-auxiliar o verbo copulativo «parecer» que, si bien permite reducir la rotundidad del desacuerdo con la consideración expuesta, no por ello oculta el compromiso afectivo de su emisor. Por otra parte, la presencia del verbo informativo «pretender» implica de nuevo por sí mismo un desacuerdo entre el escritor y la investigación original y nos orienta de la actitud del emisor del mensaje:

8. «Tampoco parecen aceptables algunas aplicaciones señaladas hace algunos años, pero hoy día cuestionadas, como el empleo del ácido ascórbico para acidificar la orina, con lo que se pretendía su indicación en la profilaxis de las infecciones urinarias, o la utilización como coadyuvante de la desferroxamina en el tratamiento de la sobrecarga crónica de hierro.»

El contenido negativo y la subjetividad del emisor los reflejan asimismo oraciones atributivas de tipo valorativo y cláusulas de actitud («es obvio»,

12 Translation of central parts of citation: We will never know whether in this editorial there is more "jealousy" than ultra-conservatism, or vice versa.

13 The proliferation of the supposed benefits of vitamin $\mathrm{C}-$ along with the all too famous failures to demonstrate its clinical efficiency in the prevention of the common cold (*) and in the cure of advanced cancer $(*)$ - has greatly contributed to its discredit (what is useful for almost everything is generally useless) 
«nos hacen dudar») — una de ellas en forma plural a pesar de ser emitida por un autor individual —, un sustantivo disfórico («disparidad») y un adjetivo entrecomillado («real»), en el siguiente ejemplo, extraído de un ED titulado «El valor de la masa ósea determinada por densitometría ósea»:

9. «Como es obvio, la disparidad en los resultados, dependientes de lo que es la matriz ósea y de lo que son los minerales óseos, nos hace dudar, independientemente de la validez de cada una de las técnicas utilizadas, de la "real" eficacia del tratamiento efectuado.»

La mitigación de la CA observada en el citado ejemplo se repite en la siguiente CA, tomada de un ED que versa sobre el empleo de medidas densitométricas para valorar la masa ósea. A la oblicuedad de esta crítica contribuyen no sólo el semi-auxiliar «parecer», sino también el matizador adverbial de probabilidad «quizás», aunque la inyección de la personalidad del autor y el fuerte compromiso adquirido subyacen en adverbios intensificadores («tremendamente», «rigurosamente», «nunca»), en un modal deóntico o de necesidad («debe») y en un pronombre personal en forma plural («nos»), todos ellos elementos que permiten reforzar la crítica y hacerla más convincente:

10. «Para concluir, nos parece tremendamente prudente el señalar que, en las medidas densitométricas actualmente realizadas, quizás hoy en día con una profusión exagerada e interpretación mal efectuada, se debe ser rigurosamente consciente de que una medida, en sí misma, nunca nos define la masa ósea de un individuo en un sentido estricto y que los cambios observados en ellas pueden depender de tantas variables (cambios en el peso, estación del año, tratamientos efectuados, zona de vivienda, hábitos alimenticios, suplementos dietéticos de minerales, etc.), que, de no tenerlos en cuenta, hacen de ellas un método carente de fiabilidad y consecuentemente de utilidad.»

Otros adjetivos, sustantivos y verbos disfóricos («fatales», «inaceptables», «peligrosas», «peligrosidad», «riesgo», «riesgos», «se agrava») caracterizan también el siguiente párrafo, tomado de un ED que trata del presente y futuro de determinados fármacos en el tratamiento de la obesidad. En este ejemplo, el verbo realizativo o performativo en plural «tenemos» ilustra la condición de experto del autor que se ve en la necesidad de informar de ciertos problemas. Por otra parte, el autor muestra su implicación afectiva mediante el uso de intensificadores adverbiales y adjetivales 
(«absolutamente», «irresponsable») y del sustantivo «prescripción» (en lugar del verbo correspondiente), que le sirve para incrementar la fuerza persuasiva de su enunciado:

11. «En este punto tenemos que llamar la atención que los extractos tiroideos son uno de los principales componentes de las peligrosas fórmulas magistrales antiobesidad de nuestro país; la mayoría de los componentes de estas fórmulas conllevan una peligrosidad potencial para el paciente, que se agrava al administrarlos en asociaciones no experimentadas, por la posibilidad de que aparezcan interacciones. De esta forma, estas formulaciones no tienen ninguna justificación en la terapéutica actual de la obesidad, y la prescripción de las mismas es absolutamente irresponsable por el riesgo que tiene el paciente de sufrir riesgos inaceptables ofatales.»

Por lo general, el(los) apellido(s) del(de los) autor(es) censurado(s) no aparece(n) en la formulación lingüística de la crítica y se sustituye(n) por números en superíndice o entre paréntesis que remiten a la bibliografía. Si en alguna ocasión se menciona al autor de forma implícita, lo que sucede en contadas ocasiones, no suele figurar en el cuerpo de la crítica sino en párrafos anteriores ${ }^{14}$.

\subsection{Articulo de Revisión (AR)}

El AR es «un estudio pormenorizado, selectivo y crítico, que integra la información esencial en una perspectiva unitaria de conjunto» (Caldeiro y otros 1993: 22). El AR consiste, pues, en la revisión o análisis de un determinado tema por un experto desde dos perspectivas: su experiencia propia y la resultante del estudio de la bibliografía publicada sobre el particular (Pulido 1989).

Con referencia a a su estructura, el AR suele constar de diferentes partes: una introducción, diversos subapartados con una discusión bibliográfica crítica o descriptiva y recopilativa, un apartado final de conclusiones con un carácter práctico que aconseja soluciones para un problema dado, unas referencias bibliográficas, tablas y figuras. El artículo puede ir firmado por uno o varios autores (el número de autores que firman los

14 Sólo en una ocasión hemos encontrado una CA directa que incluye los apellidos de los autores, pero en este caso la CA no se dirigía a ellos sino hacia el experimento que habían diseñado. 
Artículos de Revisión de nuestro corpus varía de uno a seis) y su longitud varía entre 1.288 y 10.332 palabras.

$\mathrm{Si}$ se tiene en cuenta que el volumen de la literatura biomédica se duplica cada diez años, el AR constituye una pieza de incalculable valor para muchos científicos que necesitan confiar y basarse en la información recopilada y analizada por otros. El exceso de información, a menudo compleja y contradictoria, hace que su evaluación equilibrada y profunda sea casi imposible para el inexperto. Otras veces, la barrera idiomática obliga a consultar las traducciones y valoraciones efectuadas por terceros y a creer en ellas. Por todo ello los escritores de Artículos de Revisión deben ser conocedores del tema abordado y ser capaces de filtrar el contenido de las publicaciones revisadas para así facilitar el trabajo de estar informado y al día sobre un determinado tema que, de otra forma, requeriría muchas horas de trabajo (primero una búsqueda bibliográfica y después una lectura y un análisis crítico de todos los artículos).

Los Artículos de Revisión que hemos revisado contienen gran número de críticas pero en menor grado que los Editoriales (para un análisis cuantitativo, véase nota al pie 3). Al igual que ocurría con estos últimos, su tono de voz suele ser autoritario, categórico y seguro, en tanto que reflejo de las voces de expertos, de maestros cuya experiencia les permite marcar el camino a seguir, como ilustra el ejemplo siguiente tomado de un artículo sobre la necesidad de estudios de toxicología animal necesarios para la investigación clínica y el registro de medicamentos de uso en humanos:

12. «Asombra comprobar que existen al menos 47 guías de requerimientos toxicológicos de otros tantos países. Es cada vez más evidente que la inversión económica necesaria y el nivel actual de la investigación de fármacos hacen preciso, en muchas ocasiones, un desarrollo internacional de los mismos. Del mismo modo, la comercialización debe ser multinacional, en orden a amortizar con suficiente rapidez los gastos de investigación y poder hacerlo mientras dure la patente del producto. Sin embargo, esta necesidad choca frontalmente con la variopinta situación descrita.»

Nótese la implicación afectiva y la fuerza persuasoria presentes en las formas y expresiones «asombra», «es cada vez más evidente», «hacen preciso» $\mathrm{y}$ «debe», que son un anticipo de la crítica introducida por el conector parentético «sin embargo». El autor de este AR se atreve incluso a recordar a las autoridades sanitarias cúal es su labor y a censurar un 
organismo como la FDA, aunque de forma mitigada mediante el uso de matizadores diversos («parece», «podría»):

13. «Sin embargo, parece que la FDA podría hacer peligrar dicho acuerdo, ya que recientemente hizo saber a la PMA de EE.UU. que sólo aceptará estudios de 6 meses en perros cuando las dosis empleadas sean lo suficientemente altas para caracterizar el perfil de toxicidad (incluida mortalidad) y no se detecte incremento de la toxicidad entre los estudios de 1 y 3 meses*.»

La CA contenida en los Artículos de Revisión suele tomar como blancos el afán por publicar investigaciones que muy a menudo no aportan nada nuevo a la ciencia, la ética médica o estudios incompletos y mal diseñados. Testigos de ello son los ejemplos siguientes pertenecientes a dos artículos que tratan de la intervención sobre los factores de riesgo (ejemplo 14) y protección de órganos diana (ejemplo 15):

14. «Ahora nos hemos dado cuenta de que las guías nacionales no sirven porque, aunque son similares (fruto de la copia corregida y aumentada o disminuida por el prurito de originalidad), tienen diferencias que complican la realización de un desarrollo preclínico que está internacionalmente aceptado.»

15. «Evidentemente el estudio tenía la limitación de subestimar los efectos del tabaco sobre las coronarias, al excluir aquellos que habían fallecido por CI o diabetes, que suponemos que tendrían un porcentaje más elevado de enfermedad coronaria.» 15

A propósito de ambos ejemplos, conviene recordar lo comentado en el subapartado de los Editoriales acerca de las formas flexionadas de plural. En el ejemplo 15 se puede, además, apreciar la ironía presente en el adverbio de opinión «evidentemente» y en la información adicional introducida por el verbo «suponemos».

Asimismo, la incompletez de determinados estudios obliga a no aventurarse en decisiones apresuradas, como lo refleja la CA contenida en un artículo titulado «Hiperprolactinemia patológica y FK506: ¿un inmunosupresor para los noventa?», en la cual la implicación emocional la trasmite el modificador oracional «desafortunadamente»:

15 Obviously the study underestimated the effects of tobacco on the coronary arteries as it excluded the patients who had died of diabetes. It can safely be assumed indeed that these patients would exhibit a higher percentage of coronary disease. 
16. «Desafortunadamente no se ha demostrado correlación entre nivel, dosis e incidencia de rechazo*, aunque mediante bioanálisis se ha encontrado relación entre niveles bajos de FK506 (menos de $0,3 \mathrm{ng} / \mathrm{ml}$ ) y la capacidad de crecimiento de linfocitos de biopsias hepáticas de alotrasplantes hepáticos.»

Al igual que en los Editoriales, en los Artículos de Revisión el(los) apellido(s) del(de los) autor(es) censurado(s) se menciona(n) generalmente en superíndice y raramente aparece(n) explícito(s) en la expresión de la crítica $^{16}$.

\subsection{Artículo Original (AO)}

El AO es un trabajo de investigación, realizado generalmente por varios autores de uno o de múltiples centros (en nuestra muestra lingüística, el número es de 2 a 5), que aporta informaciones de cierta relevancia sobre temas de interés general. El AO aspira a tender un puente entre el contexto y el experimento específico (general-particular) y hace hincapié sobre la ausencia o inexactitud de investigaciones anteriores, que son el motor de arranque o la causa de la investigación que se presenta. Al contrario del $\mathrm{AR}$, el AO pretende comprobar una hipótesis o una teoría, averiguar si hay algo de inexacto en ella por no haber sido corroborada, en cuyo caso intentará solucionar lo erróneo de su planteamiento o de sus conclusiones. Desde el punto de vista estructural, el AO sigue una estructura rígida, la denominada «IMRYD» («Introducción», «Materiales/Métodos», «Resultados» «Y» «Discusión»), y la longitud de los Artículos Originales revisados oscila entre 1.017 y 2.980 palabras.

Por lo general, las críticas a fuentes anteriores presentes en los Artículos Originales suelen ser indirectas, como lo ilustran los ejemplos siguientes extraídos de un artículo titulado «La trombocitopenia asociada a la infección por el virus de la inmunodeficiencia humana. Estudio inmunológico de 60 pacientes adictos a drogas por vía parenteral». De nuevo la CA se mitiga mediante el uso de modofóricos diversos, en este caso una estructura formada por un verbo copulativo en tercera persona, que contribuye a la objetivación de la crítica, y un adjetivo de probabilidad o un modal auxiliar que may be used to soften the force of an assertion or a directive (Perkins 193: 118):

16 Uno solo de todos los artículos revisados contenía dos referencias explícitas. 
17. «En el momento actual, no parece posible explicar la TP a través de un único factor etiopatogénico.»

18. «De lo contrario, pueden obtenerse conclusiones erróneas que vendrán a sumarse a las dificultades que conlleva el estudio de la especificidad de los autoanticuerpos antiplaquetarios y la interpretación de los resultados obtenidos con estas técnicas*.»

A diferencia de lo que ocurre en la CA tomada de los Editoriales y Artículos de Revisión, en muchas de las CCAA incluidas en los Artículos Originales se produce una desfocalización del autor, que pasa a un segundo plano en lo aseverado en el enunciado, y la crítica se atribuye a un sujeto no personal, como lo refleja la siguiente CA tomada de un artículo que versa sobre receptores estrogénicos y fibroadenomas mamarios:

19. «Así, nuestros resultados confirman la opinión de que la aparición de la neuropatía diabética no se relaciona exclusivamente con la edad del paciente*, sino también con la duración de la enfermedad.»

La ocultación del autor, que sirve para protegerse de los posibles ataques o contrapropuestas del público destinatario, también alcanza a las autocríticas, muy comunes en los Artículos Originales, como lo ilustra el ejemplo siguiente extraído de un AO que trata de la infección hospitalaria por enterococos:

20. «Sin embargo, el uso exclusivo de cefalosporinas de amplio espectro, quizás el factor predisponente para la sobreinfección enterocócica mejor reconocido y denunciado*, tuvo un papel secundario en el presente trabajo.»

La crítica hacia lagunas conceptuales, teóricas o prácticas, que se pretende llenar, es también una característica omnipresente en los Artículos Originales, como lo testifica la siguiente CA presente en un artículo sobre la prevalencia de los estados de hipercoagulabilidad en pacientes con trombosis:

21. «Si bien se conoce la estrecha relación entre determinadas situaciones clínicas tales como cirugía, sepsis, neoplasis y la aparición de trombosis, son escasos los estudios que han analizado simultáneamente un amplio abanico de alteraciones hereditarias y adquiridas causantes de estados de hipercoagulabilidad en pacientes con trombosis.» 
Se pueden asimismo censurar, de forma matizada, creencias afianzadas, como lo ejemplifica la siguiente CA procedente de un artículo sobre la fiebre botonosa mediterránea:

22. «El corazón participa con mayor frecuencia de lo indicado en libros y publicaciones: habituales arritmias, pericarditis y esencialmente miocardiopatía, edema pulmonar cardiogénico (en ocasiones no cardiogénico).»

Son también frecuentes las expresiones de escepticismo que los autores de las críticas utilizan para poner límites a sus conocimientos por una parte y, por otra, para reducir su grado de compromiso personal, como lo ilustra la siguiente CA tomada del citado artículo sobre receptores estrogénicos y fibroadenomas mamarios:

23. «Otro aspecto no estudiado previamente, al menos en nuestro conocimiento es la presencia de RE* en estos FAD asociados a carcinoma.»

Por último, cabe reseñar que sólo en una ocasión hemos encontrado una CA dirigida a los resultados incompletos de un experimento en la que se menciona de forma explícita el apellido de los autores reprobados, como se aprecia en el siguiente ejemplo extraído de un artículo titulado «Características epidemiológicas de la brucelosis en el distrito de atención primaria de salud Sierra de Cádiz»:

24. «En nuestro estudio desglosamos el riesgo de cada producto lácteo que se consume, pero salvo casos aislados entre los que están Cueto Espinar et al*, que obtienen un $85 \%$ de consumo de leche y un $30 \%$ de consumo de queso sin higienizar, y Rodríguez Martín*, que pone de manifiesto un $72 \%$ para la leche y un $45,14 \%$ para el queso, la mayor parte de los autores ofrecen cifras globales sobre el consumo de productos lácteos sin higienizar, destacando Colmenero et al* con un 70\%; Arribas Llorente et al*, con un 54,5\%; Ariza Cardenal*, un 60\%; Taylor y Perdue*, un 67\%; Nogales Pérez et al*, un $90 \%$, y Talamante Serrulla et al*, con un $39,15 \%$ de casos que consumen productos lácteos sin garantías sanitarias.»

\subsection{Notas Clínicas (NC)}

A pesar de que las revistas biomédicas generales son cada vez más reacias a publicar casos aislados en forma de Notas Clínicas, lo cierto es que siguen publicándose, sobre todo en revistas de especialidad, y su interés reside en su propia naturaleza y no en la discusión ni en la revisión de la 
literatura o en las hipótesis de cualquier tipo que puedan elaborarse a partir de ella. Las Notas Clínicas suelen informar sobre fenómenos aislados, como dejan bien patentes los títulos de algunos artículos incluidos en nuestro corpus: «Síndrome maligno por neurolépticos en el anciano: descripción de cuatro pacientes», «cardiopatía e infección por el virus de la inmunodeficiencia humana: presentación de dos casos», etc.

Ello, sin embargo, no implica que una NC no contenga críticas, aunque su frecuencia es sensiblemente inferior a la un ED, un AR o un AO. Por otra parte, la CA recopilada en las Notas Clínicas comparte rasgos retóricos comunes con la $\mathrm{CA}$ registrada en los articulos originales, es decir, suele aparecer en la introducción, justificando por tanto el interés de la publicación del caso, o en la secciones «Comentarios» $\mathrm{y}$ «Discusión», y se refiere mayoritariamente a desórdenes que nunca se han comunicado en determinados contextos o a lagunas informativas sobre temas dados en la literatura, como lo refleja el ejemplo siguiente:

25. «Esta infección ha sido ampliamente descrita en adultos infectados por $\mathrm{VIH}^{*}$; sin embargo, existen muy pocos trabajos publicados de esta infección oportunista en niños, siendo uno de ellos muy superponible al caso que aquí se presenta, con un curso fulminante en un corto periodo de tiempo*.»

A veces la CA se dirige hacia diseños erróneos en determinados estudios o tratamientos que ocasionan resultados cuestionables, y su tono suele estar suavizado por matizadores diversos:

26. «Sin embargo, estos autores no ajustaron sus resultados en función de la edad, el sexo, la raza y el hábito tabáquico, lo cual limita considerablemente la validez de sus estimaciones*.»

27. «No parece probable que la asociación de corticoides en el tratamiento de esta paciente haya favorecido una recaída, ya que, aunque se ha sugerido que el uso de corticoides podría interferir en la penetración de antibióticos en el LCR por la disminución de permeabilización de la barrera hematoencefálica*, ello no ha sido demostrado* y podría, en todo caso, resultar en una reagudización o en el retraso en la esterilización del líquido*.»

\section{Conclusiones}

En general puede considerarse que los artículos se dividen en dos grandes bloques: por un lado, aquéllos que la misma revista encarga a los autores (Editoriales y Revisiones, que se enmarcan dentro de la denominada 
«formación médica continuada») y, por otro, aquéllos que los autores realizan de forma espontánea y envían a las revistas para su publicación (Artículos Originales y Notas Clínicas). Esta distinción inicial ha facilitado la consecución de los objetivos fijados en este estudio: 1) relacionar las variaciones observadas en las formulaciones lingüísticas elegidas para transmitir el desacuerdo profesional con la función comunicativa específica de los cuatro géneros analizados; 2) examinar su nivel de pretensión científica; 3) analizar la relación existente entre autor y audiencia.

Abordemos, en primer lugar, los Editoriales. Las revistas suelen solicitarlos a personas que no forman parte del Comité Editorial, basándose en su categoría científica o en su experiencia sobre un tema específico, y recomendarles que den su visión personal al respecto y orienten la investigación futura en determinado sentido. No es, por tanto, de extrañar que se note en ellos una fuerte presence of authorial persona (Atkinson 1996: 359) y que su escritor tienda a adoptar una position of authority (Korhonen y Kush 1989: 74) frente a sus lectores, precisamente por su condición de experto dentro de su comunidad. Esa condición de experto es la que le va a permitir evaluar la investigación científica con conocimiento de causa, cuestionar investigaciones anteriores de forma directa, incluso agresiva y ofensiva, a veces condescendiente, irónica y sarcástica, para intentar convencer al lector de la exactitud y la validez de sus aseveraciones. En este sentido, el ED se encuadra dentro del discurso argumentativo/evaluativo/persuasivo y es similar al ensayo, en tanto que interpretación personal e individual de un determinado tema (Sager y otros 1980). En definitiva, el ED es discourse on discourse (Vihla 1999: 17).

Dentro del mismo discurso argumentativo, evaluativo e incluso persuasivo, se encuadra el Artículo de Revisión, cuyo autor, por su calidad de experto con una posición consolidada, tiene la función de evaluar de forma crítica y exhaustiva estudios previamente publicados, con el fin de suministrar guías prácticas sólidas a un público muy amplio, que comprende médicos generales y especialistas, médicos posgraduados y estudiantes de medicina, además de otros potenciales lectores, puesto que los temas que trata pueden también tener conexiones con varias especialidades.

A diferencia del Editorial y del Artículo de Revisión, el Artículo Original pertenece más al discurso informativo que al evaluativo/argumentativo y la pretensión científica que presenta su autor debe aún pasar por 
el tamiz de la comunidad científica. Para no provocar posibles convulsiones a consecuencia de sus reivindicaciones, el escritor de un AR no se atreverá, por interés propio y también por interés de la institución en la que trabaja, a censurar a sus colegas en un tono demasiado duro y directo sino que recurrirá a un lenguaje aproximativo, característico de la negociación dentro de la comunicación social. Así sus críticas se formularán de modo oblicuo, es decir, estarán llenas de rodeos (modales, adverbios de probabilidad, expresiones de escepticismo) para evitar que se cierren las puertas de las editoriales que pondrán su producto intelectual en el mercado.

En cuanto a las Notas clínicas, pertenecen sobre todo al discurso informativo y de los cuatro géneros considerados en este estudio, son las menos argumentativas y las que presentan el nivel más bajo de pretensión científica. Su situación comunicativa, pues, no suele favorecer el debate científico.

Nuestro estudio ha puesto entonces de manifiesto que las diferencias observadas en la práctica discursiva de las críticas recopiladas reflejan diferentes funciones comunicativas que, a su vez, determinan los papeles sociales de los escritores y les dictan patrones discursivos determinados. En este sentido, creemos que los resultados de nuestra investigación están en consonancia con los de otros autores que se han ocupado de este mismo tema, aunque en lenguas diferentes al español (Myers 1990; Swales 1990; Salager-Meyer 2001) y corroboran su percepción de las relaciones existentes entre la elección del lenguaje y la construcción social del texto. Confiamos, además, en que el análisis cuantitativo que estamos llevando a cabo servirá para consolidar las tendencias cualitativas que hemos observado en este estudio, y esperamos haber contribuido, siguiendo a Caldeiro y otros (1993: IX), a conferir al español «rango de lengua científica».

\section{Referencias bibliográficas}

Ahmad, U. K. (1997). Research article introductions in Malay: rhetoric in an emerging research community. En Duszak, A. (Ed.): op. cit.: 273-303.

Alcaraz Ariza, M. A. \& F. Salager-Meyer (2002). La disensión (¿directa o velada?) en la prosa médica española (1930-1999). En Lebende Sprachen 4: 180-185.

Álvarez-Dardet C.; F. Bolúmar \& M. Porta-Serra (1987). Tipos de estudios. En Medicina Clínica 89: 296-301. 
Atkinson, D. (1996). The philosophical transactions of the Royal Society of London. 1675-1975: A sociohistorical discourse analysis. En Language and Society 25 (3): 331-371.

Bailar, J.; Louis, T. A.; Lavori, P. W. \& M. Polansky (1984). A classification for biomedical research reports. En The New England Journal of Medicine 31: 1.482-1.487.

Bazerman, C. (1998). Shaping Written Knowledge: The Genre and Activity of the Experimental Article in Science. Madison (Wisconsin): University of Wisconsin Press.

Belcher, D. (1995). Writing critically across the curriculum. En Belcher, D. \& G. Braine (Eds.). En Academic Writing in a Second Language. Essays on Research and Pedagogy. Norwood (N. J.): Ablex Publishing. 133-155.

Bhatia, V.K. 1993. Analysing Genre. London: Longman.

-, (1998): Genre-mixing in academic introductions. En English for Specific Purposes 16 (3): 181-193.

Biber, D. (1988). Variation across Speech and Writing. New York: CUP.

Bloch, J. \& L. Li (1995). A comparison of the use of citations in Chinese and English academic discourse. En Belcher, D. \& G. Braine (Eds.). Academic Writing in a Second Language. Essays on Research and Pedagogy. Norwood (N. J.): Ablex Publishing. 231-277.

Caldeiro, M. ${ }^{a}$ A.; Feliu, E.; Foz, M.; Gracia, D.; Herranz, G.; Lience, E.; Pulido, M.; Ribera, M. a ; Rey-Joly, C.; Ruiz, F. J. \& O. Vilarroya (1993). Manual de Estilo. Publicaciones biomédicas. Barcelona: Mosby/Doyma.

Chafe, W. (1982). Integration and involvement in speaking, writing and oral literature. En Tannen, D. (Ed.). Spoken and Written Language: Exploring Orality and Literacy (Advances in Discourse Processes 4). Norwood (N. J.): Ablex Publishing. 35-54.

Chubin, D. E. \& S. D. Moitra (1975). Content analysis of references: adjunct or alternative to citation counting. En Social Studies of Science 5 (4): 15-28.

Connor, U. (1996). Contrastive rhetoric: cross-cultural aspects of second-language writing. Cambridge: CUP.

Devitt, A. (1991). Intertextuality in text-accounting: generic, referential and functional. En Bazerman, C. \& J. Paradis (Eds.). Textual Dynamic in the Professions: Historical and Contemporary Writing in Professional Communities. Madison (Wisconsin). University of Wisconsin Press. 336-358.

Do, Q. T. (1989). Notes on education in the traditional culture of Vietnam. En Journal of Vietnamese Studies 1 (2): 13-17.

Dudley-Evans, T. \& M. J. St John (1998). Developments in English for Specific Purposes. A Multidisciplinary Approach. Cambridge: CUP.

Duszak, A. (Ed.) (1997). Culture and Styles in Academic Discourse. Berlin: Mouton de Gruyter. 
Farrell, L. (1997). Doing well ... doing badly: an analysis of the role of conflicting cultural values in judgements of relative academic achievement. En Duszak, A. (Ed.). op. cit.: 63-89.

Ferguson, G. (2001). If you pop over there: a corpus-based study of conditionals in medical discourse. En English for Specific Purposes 20 (1): 61-82.

Freedman, A. \& P. Medway (Eds.) (1994). Genre and the New Rhetoric. London: Taylor $\&$ Francis.

Fye, W. B. (1987). The literature of American internal medicine: A historical view. En Annals of Internal Medicine 106: 451-460.

Grabe, W. \& R. Kaplan (1997). On the writing of science and the science of writing: hedging in science texts and elsewhere. En Markkanen, A. \& H. Schröder (Eds.). Hedging and Discourse: Approaches to the analysis of a Pragmatic Phenomenon in Academic Texts. Berlin: Mouton de Gruyter. 151-168.

Hemais, B. (2001). The discourse of research and pactice in marketing journals. En English for Specific Purposes 20 (1): 57-58.

Huckin, T. N. \& T. Olsen (1984). On the use of informants in LSP discourse analysis. En Pugh, A. \& J. Ulijn (Eds.). Reading for Professional Purposes. London: Heinemann. 120-129.

Hunston, S. (1993). Professional conflict: disagreement in academic discourse. En Baker, M.; Francis, G. \& E. Tognini-Bonelli (Eds.). Texts and Technology. In Honor of John Sinclair. Amsterdam: John Benjamins. 115-134.

Hutchinson, T. \& A. Waters (1987). English for Specific Purposes: A Learner-Centered Approach. Cambridge: CUP.

Hyland, K. (2000). Disciplinary Discourses: Social Interactions in Academic Writing. London: Longman.

Korhonen, R. \& M. Kush (1989). The rhetorical funcion of the first person in philosophical texts - the influence of intellectual style, paradigm and language — . En Kush, M; Martin, D. \& S. Stirred (Eds.). Text Interpretation and Argumentation. Hamburg: Buske. 61-78.

Kourilova, M. (1996). Interactive function of language in peer reviews of medical papers written by NN users of English. En UNESCO-ALSED LSP Newsletter 19 (1): 4-21.

Kress, G. (1986). Reading, Writing and Power. En AALA. Occasional Papers 9: 98-117.

López López, P. (1996). Bibliometría: la medida de la información. En López Yepes, J. (Coord.). Manual de Información y Documentación. Madrid: Ediciones Pirámide. 488-512.

López Piñero, J. M.; Navarro, V. \& E. Portela (1989). La Revolución Científica. Madrid: Historia, 16.

MacRoberts, M. \& B. MacRoberts (1984). The negational reference or the art of dissembling. En Social Studies of Science 14: 91-94. 
Moravcsik, M. \& P. Murugesan (1975). Some results on the function and quality of citations. En Social Studies of Science 5 (1): 45-53.

Motta-Roth. D. (1998). Discourse analysis and aacademic book reviews: a study of text and disciplinary cultures. En Fortanet, I.; Posteguillo, S.; Palmer, J. C. \& J. F. Coll (Eds.). Genre Studies in English for Academic Purposes. Castellón: Publicaciones de la Universitat Jaume I. 29-59.

Mulrow, C. D. (1987). The medical review article: state of the science. En Annals of Internal Medicine 106: 485-488.

Myers, G. (1989). The pragmatic of politeness in scientific articles. En Applied Linguistics 10: $1-35$.

Nguyen, X. T. (1988). Understanding Vietnamese Students: a focus on their passive attitude. En Journal of Vietnamese Studies 1 (1): 19-25.

North, S. (1992). On book reviews in rhetoric and composition. En Rhetoric Review 10: 348-363.

Paltridge, B. (1997). Genre, Frames and Writing in Research Settings. Amsterdam: John Benjamins.

Perkins, M. (1983). Modal expressions in English. London: Frances Pinter.

Posteguillo, S. (2000). A comparative genre analysis of computer science English: contending rhetorics. En The ESPecialist 21 (1): 43-69.

Pulido, M. (1987). El editorial. En Medicina Clínica 92: 413-414.

-, (1989). El artículo de revisión. En Medicina Clínica 93: 745-746.

Sager, J. C.; Dungworth, D. \& P. F. McDonald (1980). English Special Languages: Principles and Practice in Science and Technology. Wiesbaden: Brandstetter.

Salager-Meyer, F. (1999). Contentiousness in medical English written discourse: a diachronic study (1810-1995). En TEXT 19 (3): 371-398.

-, (2000). Rhetorical evolution of oppositional discourse in French academic writing. En HERMES 25: 23-48.

-, (2001). From self-highlightedness to self-effacement: a genre-based study of the sociopragmatic function of criticism in medical discourse. En LSP and Professional Communication 1 (2) : 63-84.

Salager-Meyer, F. \& N. Zambrano (2001). The bittersweet rhetoric of controversiality in 19th- and 20th-century French and English medical literature. En Journal of Historical Pragmatics 2 (1): 141-173.

Salager-Meyer, F. \& M. A. Alcaraz Ariza (2001). Lo cortés no quita lo valiente: la retórica de la discrepancia en el discurso médico escrito en español (1880-1899). En Palmer, J. C.; Posteguillo, S. \& I. Fortanet (Eds.). Discourse analysis and terminology in Languages for Specific Purposes. Collecció Estudis Filològics 5. Castellón: Publicaciones de la Universitat Jaume I. 15-24.

Schramm, A. (1996). Using aspects to express viewpoints in EST texts. En English for Specific Purposes 15 (2): 141-164. 


\section{6}

Skrabanek, P. \& J. McCormick (1992). Sofismas y desatinos en medicina. Barcelona: Doyma.

Swales, J. (1990). Genre Analysis. English in Academic and Research Settings. Cambridge: CUP.

Swales, J. \& C. Feak (1995). From information transfer to data commentary. En TESOL France Journal 2 (2): 79-93.

Taylor, G. \& T. G. Chen (1991). Linguistic, cultural and sub-cultural issues in contrastive discourse analysis: Anglo-American and Chinese scientific texts. En Applied Linguistics 12: 319-336.

Trimble, L. (1985). English for Science and Technology: A Discourse Approach. Cambridge: CUP.

Valle, E. (1999). A Collective Intelligence: The Life Sciences in the Royal Society as a Scientific Discourse Community. 1665-1965. En Anglicana Turkuensia 17.

Vassileva, I. (2001). Commitment and detachment in English and Bulgarian academic writing. En English for Specific Purposes: An International Journal 20 (1): 83-102.

Vihla, M. (1999). Medical Writing. Modality in Focus. Amsterdam: Rodopi.

Widdowson, H. (1983). Learning Purpose and Language Use. Oxford: OUP.

Zambrano, N. (1999). Las confrontaciones académicas (CCAA) en el discurso médico escrito en inglés y francés: un estudio diacrónico comparativo(1810-1995). Memoria de Maestría inédita. Mérida (Venezuela): Universidad de Los Andes. 